\title{
Generation of Interface for an Allen-Cahn Equation with Nonlinear Diffusion
}

\author{
M. Alfaro ${ }^{1}$ and D. Hilhorst ${ }^{2 *}$ \\ ${ }^{1}$ I3M, Université de Montpellier 2, CC051, Place Eugène Bataillon, \\ 34095 Montpellier Cedex 5, France. \\ ${ }^{2}$ CNRS et Laboratoire de Mathématiques, Université de Paris-Sud 11, \\ 91405 Orsay Cedex, France.
}

\begin{abstract}
In this note, we consider a nonlinear diffusion equation with a bistable reaction term arising in population dynamics. Given a rather general initial data, we investigate its behavior for small times as the reaction coefficient tends to infinity: we prove a generation of interface property.
\end{abstract}

Key words: degenerate diffusion, singular perturbation, motion by mean curvature, population dynamics.

AMS subject classification: 35K65, 35B25, 35R35, 92D25.

\section{Introduction}

We consider the degenerate parabolic problem

$$
\left(P^{\varepsilon}\right) \begin{cases}u_{t}=\Delta\left(u^{m}\right)+\frac{1}{\varepsilon^{2}} f(u) & \text { in } Q_{T}:=\Omega \times(0, T) \\ \frac{\partial\left(u^{m}\right)}{\partial \nu}=0 & \text { on } \partial \Omega \times(0, T) \\ u(x, 0)=u_{0}(x) & \text { in } \Omega,\end{cases}
$$

with $\varepsilon>0$ a small parameter. Here $\Omega$ is a smooth bounded domain in $\mathbb{R}^{N}(N \geq 2), \nu$ is the Euclidian unit normal vector exterior to $\partial \Omega$ and $m \geq 2$.

\footnotetext{
*Corresponding author. E-mail: danielle.hilhorst@math.u-psud.fr
} 
We assume that $f$ is smooth and has exactly three zeros $0<a<1$ such that

$$
f^{\prime}(0)<0, \quad f^{\prime}(a)>0, \quad f^{\prime}(1)<0 .
$$

Moreover we suppose that the initial function $u_{0} \in C^{2}(\bar{\Omega})$ is nonnegative, with support

$$
\text { Supp } u_{0}:=\left\{x \in \Omega \mid u_{0}(x)>0\right\} \subset \subset \Omega \text {. }
$$

Furthermore we define the initial interface $\Gamma_{0}$ by

$$
\Gamma_{0}:=\left\{x \in \Omega \mid u_{0}(x)=a\right\},
$$

and suppose that $\Gamma_{0}$ is a smooth hypersurface without boundary such that

$$
\begin{aligned}
& \Gamma_{0} \subset \subset \Omega \quad \text { and } \quad \nabla u_{0}(x) \neq 0 \quad \text { if } x \in \Gamma_{0}, \\
& u_{0}>a \quad \text { in } \quad \Omega_{0}^{(1)}, \quad u_{0}<a \quad \text { in } \Omega_{0}^{(0)},
\end{aligned}
$$

where $\Omega_{0}^{(1)}$ denotes the region enclosed by $\Gamma_{0}$ and $\Omega_{0}^{(0)}$ the region enclosed between $\partial \Omega$ and $\Gamma_{0}$.

We prove a generation of interface property, namely that the solution $u^{\varepsilon}$ quickly becomes close to 1 or 0 , except in an $\mathcal{O}(\varepsilon)$ neighborhood of the initial interface $\Gamma_{0}$, creating a steep transition layer around $\Gamma_{0}$. More precisely, we are in presence of the following phenomenon: in the very early stage, the nonlinear diffusion term is negligible when compared with the reaction term $\varepsilon^{-2} f(u)$. Hence, under the rescaling in time $\tau=t / \varepsilon^{2}$, the equation is well approximated by the ordinary differential equation $u_{\tau}=f(u)$. In view of the bistable nature of $f, u^{\varepsilon}$ quickly approaches the stable equilibria of the ordinary differential equation, 0 or 1 , and an interface is formed between the regions $\left\{u^{\varepsilon} \approx 0\right\}$ and $\left\{u^{\varepsilon} \approx 1\right\}$.

The organization of this note is as follows. In Section 2. we briefly explain how Problem $\left(P^{\varepsilon}\right)$ arises in population dynamics. In Section 3., we recall known results about the well-posedness of Problem $\left(P^{\varepsilon}\right)$ and a comparison principle. In Section 4., we prove the generation of interface property for Problem $\left(P^{\varepsilon}\right)$. To that purpose we construct sub- and super-solutions by modifying the solution of the corresponding ordinary differential equation $u_{t}=\varepsilon^{-2} f(u)$. We also show the optimality of the generation time $t^{\varepsilon}:=f^{\prime}(a)^{-1} \varepsilon^{2}|\ln \varepsilon|$ and prove that the thickness of the interface is of order $\mathcal{O}(\varepsilon)$ at the generation time $t^{\varepsilon}$. Our method of proof follows the same lines as that of [2] and [1]. It is slightly different from those of Xinfu Chen [5] and [6], who transforms the reaction function $f$. We postpone to future work the study of the interface motion after the generation time of the interface.

Finally let us mention articles involving the singular limit of reaction-diffusion equations with nonlinear diffusion. Feireisl [8] studies the singular limit of a degenerate parabolic equation in the whole space $\mathbb{R}^{N}$. He studies the problem in the scaling

$$
u_{t}=\varepsilon \Delta\left(u^{m}\right)+\frac{1}{\varepsilon} f(u)
$$


where, in the limit $\varepsilon \rightarrow 0$, the limit free boundary moves according to motion by constant speed (that of a related traveling wave). In a similar scaling, Hilhorst, Kersner, Logak and Mimura [11] investigate the singular limit of this equation in a bounded domain with a monostable reaction term. In both of these papers, they prove that the solution $u^{\varepsilon}$ of the nonlinear diffusion equation converges to 0 or 1 on both sides of an interface moving with constant normal velocity. In this scaling, proofs about the interface motion can be performed with using only one term in the asymptotic expansion whereas we would need to use two terms in the case of Problem $\left(P^{\varepsilon}\right)$ as well as a suitable linearization procedure; this is far from trivial here since Problem $\left(P^{\varepsilon}\right)$ is degenerate parabolic.

\section{The biological context}

In this section, we discuss nonlinear diffusion in population dynamics. It is well-known that the control of a population can be achieved by introducing density dependent birth or death rates. In [9], Gurney and Nisbet show that the introduction of a nonlinearity into the dispersal behavior of a species - which behaves in an otherwise linear way - can, in an inhomogeneous environment, lead to a regulatory effect. More precisely, they consider the equation

$$
u_{t}=-\operatorname{div} \mathbf{j}+G(x) u,
$$

where $u(x, t)$ denotes the population density, $G=G(x)$ the growth function only depending on the location and $\mathbf{j}(x, t)$ the local population current density. By using the well-known random motion model one obtains the linear equation $u_{t}=\Delta u+G(x) u$. Another possibility is to choose the biased random motion model where movements are largely random but slightly modified by the distribution of the fellows; the corresponding equation is then written as $u_{t}=\Delta u+\operatorname{div}(u \operatorname{grad} u)+$ $G(x) u$. Nevertheless, Carl [4] has observed that arctic ground squirrels migrate from densely populated areas into sparsely populated ones, even when the latter is less favorable (burrow sites not available, intensive predation). For such species, migration to avoid crowding, rather than random motion, is the primary cause of dispersal. To describe such movements, Gurney and Nisbet use the directed motion model where individuals can only stay put or move down the population gradient; this model yields the degenerate parabolic equation

$$
u_{t}=\Delta\left(u^{2}\right)+G(x) u \text {. }
$$

In [9], the authors perform a qualitative analysis of the three different dispersal models (random motion, biased random motion and directed motion). They conclude that the introduction of density dependent diffusion is efficient to study the dynamics of a population which regulates its size below the carrying capacity set by the supply of nutrients.

Gurtin and Mac Camy [10] proposed the class of equations which we study here and which involves degenerate diffusion and nonlinear reaction, namely

$$
u_{t}=\Delta\left(u^{m}\right)+f(u), \quad m \geq 2 .
$$


In absence of a reaction term, equation (2.2) reduces to the so-called porous medium equation

$$
u_{t}=\Delta\left(u^{m}\right)
$$

which describes, among others, the flow of an ideal gas in a homogeneous medium $(m \geq 2)$, groundwater infiltration (Boussinesq's equation, $m=2$ ), the spread of a thin viscous film under gravity $(m=4)$, and thermal propagation in plasma $(m \simeq 6)$. The porous medium equation has been extensively investigated in literature: we refer to the book of Vásquez [12] and the references therein. The main feature of these equations is that they degenerate at the points where $u=0$. As a consequence, a loss of regularity of solutions occurs and disturbances propagate with finite speed. This phenomenon contrasts with the infinite speed of propagation of solutions of the heat equation $u_{t}=\Delta u$.

\section{Comparison principle and well-posedness}

Since the diffusion term degenerates at the points where $u^{\varepsilon}=0, u^{\varepsilon}$ is not smooth. This leads us to define a notion of weak solution for Problem $\left(P^{\varepsilon}\right)$, in a similar way as it is done by Aronson, Crandall and Peletier [3] for a corresponding one-dimensional problem.

Definition 1. A function $u^{\varepsilon}:[0, \infty) \rightarrow L^{1}(\Omega)$ is a weak solution of Problem $\left(P^{\varepsilon}\right)$ if, for all $T>0$,

(i) $u^{\varepsilon} \in C\left([0, \infty) ; L^{1}(\Omega)\right) \cap L^{\infty}\left(Q_{T}\right)$;

(ii) $u^{\varepsilon}$ satisfies the integral equality

$$
\begin{aligned}
\int_{\Omega} u^{\varepsilon}(T) \varphi(T)-\iint_{Q_{T}}\left(u^{\varepsilon} \varphi_{t}+\left(u^{\varepsilon}\right)^{m} \Delta \varphi\right)=\int_{\Omega} u_{0} \varphi(0) & \\
& +\iint_{Q_{T}} \frac{1}{\varepsilon^{2}} f\left(u^{\varepsilon}\right) \varphi
\end{aligned}
$$

$$
\text { for all } \varphi \in C^{2}\left(\overline{Q_{T}}\right) \text { such that } \varphi \geq 0 \text { and } \frac{\partial \varphi}{\partial \nu}=0 \text { on } \partial \Omega \text {. }
$$

A sub-solution (respectively a super-solution) of Problem $\left(P^{\varepsilon}\right)$ is a function satisfying (i) and (ii) with equality replaced by $\leq$ (respectively $\geq$ ).

Theorem 2 (Existence and comparison principle). Let $T>0$ be arbitrary. The following properties hold:

(i) Let $u^{-}$and $u^{+}$be a sub-solution and a super-solution of Problem $\left(P^{\varepsilon}\right)$ with initial data $u_{0}^{-}$ and $u_{0}^{+}$respectively.

$$
\text { If } u_{0}^{-} \leq u_{0}^{+} \text {then } u^{-} \leq u^{+} \text {in } Q_{T} \text {; }
$$


(ii) Problem $\left(P^{\varepsilon}\right)$ has a unique weak solution $u^{\varepsilon}$ which is such that

$$
0 \leq u^{\varepsilon} \leq \max \left(1,\left\|u_{0}\right\|_{L^{\infty}(\Omega)}\right) \text { in } Q_{T} ;
$$

(iii) $u^{\varepsilon} \in C\left(\overline{Q_{T}}\right)$.

The proof of Theorem 2 is standard; it can be performed by using the same lines as that of Theorem 5 in [3]. The continuity of $u^{\varepsilon}$ follows from [7].

The following result turns out to be an essential tool when constructing smooth sub- and supersolutions of Problem $\left(P^{\varepsilon}\right)$.

Lemma 3. Let $u^{\varepsilon}$ be a continuous nonnegative function in $\bar{\Omega} \times[0, T]$. Define $\Omega_{t}^{\text {supp }}=\{x \in$ $\left.\Omega \mid u^{\varepsilon}(x, t)>0\right\}$ and $\Gamma_{t}^{\text {supp }}=\partial \Omega_{t}^{\text {supp }}$ for all $t \in[0, T]$. Suppose that the family $\Gamma:=\cup_{0<t \leq T} \Gamma_{t}^{\text {supp }} \times$ $\{t\}$ is sufficiently smooth and let $\nu_{t}^{\text {supp }}$ be the outward normal vector on $\Gamma_{t}^{\text {supp }}$. Suppose moreover that

(i) $\nabla\left(u^{\varepsilon}\right)^{m}$ is continuous in $\bar{\Omega} \times[0, T]$;

(ii) $\mathcal{L}\left[u^{\varepsilon}\right]:=u_{t}^{\varepsilon}-\Delta\left(u^{\varepsilon}\right)^{m}-\frac{1}{\varepsilon^{2}} f\left(u^{\varepsilon}\right)=0$ in $\{(x, t) \in \bar{\Omega} \times[0, T]$ such that $\left.u^{\varepsilon}(x, t)>0\right\}$;

(iii) $\frac{\partial\left(u^{\varepsilon}\right)^{m}}{\partial \nu_{t}^{\text {supp }}}=0$ on $\partial \Omega_{t}^{\text {supp }}$, for all $t \in[0, T]$.

Then $u^{\varepsilon}$ is a solution of Problem $\left(P^{\varepsilon}\right)$. Similarly $u$ is a sub-solution (respectively a super-solution) of Problem $\left(P^{\varepsilon}\right)$ if the equality in (ii) is replaced by $\leq$ (resp. $\geq$ ) and if the equality in (iii) is replaced by $\leq($ resp. $\geq)$.

We refer to [11] for the proof.

\section{Generation of interface}

In this section we prove that, given a nearly arbitrary initial function $u_{0}$, the solution $u^{\varepsilon}$ quickly becomes close to 1 or 0 , except in an $\mathcal{O}(\varepsilon)$ neighborhood of the initial interface $\Gamma_{0}$, creating a steep transition layer around $\Gamma_{0}$. The time needed to develop such a transition layer is of order $\mathcal{O}\left(\varepsilon^{2}|\ln \varepsilon|\right)$.

Theorem 4 (Generation of interface). Assume $m \geq 2$. Let $\gamma \in(0, \min (a, 1-a))$ be arbitrary and define $\mu$ as the derivative of $f(u)$ at the unstable equilibrium $u=a$, namely

$$
\mu=f^{\prime}(a) \text {. }
$$

Moreover, set

$$
t^{\varepsilon}:=\mu^{-1} \varepsilon^{2}|\ln \varepsilon| .
$$

Then there exist positive constants $\varepsilon_{0}$ and $M_{0}$ such that, for all $\varepsilon \in\left(0, \varepsilon_{0}\right)$, 
(i) for all $x \in \Omega$, we have that

$$
0 \leq u^{\varepsilon}\left(x, t^{\varepsilon}\right) \leq 1+\gamma ;
$$

(ii) for all $x \in \Omega$ such that $\left|u_{0}(x)-a\right| \geq M_{0} \varepsilon$, we have that

$$
\begin{aligned}
& \text { if } u_{0}(x) \geq a+M_{0} \varepsilon \text { then } u^{\varepsilon}\left(x, t^{\varepsilon}\right) \geq 1-\gamma, \\
& \text { if } u_{0}(x) \leq a-M_{0} \varepsilon \text { then } u^{\varepsilon}\left(x, t^{\varepsilon}\right) \leq \gamma .
\end{aligned}
$$

Theorem 4 will be proved by constructing a suitable pair of sub- and super-solutions. As mentioned above, the nonlinear diffusion term is negligible in this early stage so that the behavior of the solution is governed by the ordinary differential equation $u_{t}=\frac{1}{\varepsilon^{2}} f(u)$.

An immediate consequence of Theorem 4 is that $u^{\varepsilon}\left(x, t^{\varepsilon}\right)$ is close to 0 or 1 , except in an $\mathcal{O}(\varepsilon)$ neighborhood of the initial interface $\Gamma_{0}$. In other words, the transition layers which have developed have an $\mathcal{O}(\varepsilon)$ thickness.

Corollary 5 (Thickness of the transition layers at time $\left.t^{\varepsilon}\right)$. Let $\eta \in(0, \min (a, 1-a))$ be an arbitrary constant. Then there exist positive constants $\varepsilon_{0}$ and $\mathcal{C}$ such that, for all $\varepsilon \in\left(0, \varepsilon_{0}\right)$,

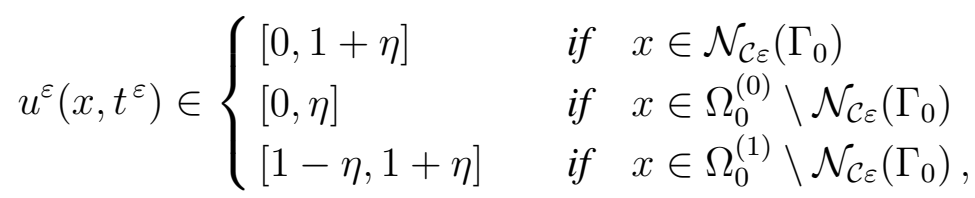

where $\Omega_{0}^{(1)}$ denotes the region enclosed by $\Gamma_{0}, \Omega_{0}^{(0)}$ the region enclosed between $\partial \Omega$ and $\Gamma_{0}$, and

$$
\mathcal{N}_{r}\left(\Gamma_{0}\right):=\left\{x \in \Omega, \operatorname{dist}\left(x, \Gamma_{0}\right)<r\right\}
$$

denotes the r-neighborhood of $\Gamma_{0}$.

We will also show that the generation time $t^{\varepsilon}:=\mu^{-1} \varepsilon^{2}|\ln \varepsilon|$ is optimal. In other words, the interface is not fully developed until $t$ becomes close to $t^{\varepsilon}$. More precisely, the following result holds.

Proposition 6. Denote by $t_{\text {min }}^{\varepsilon}$ the smallest time such that (4.4) holds. Then there exists a constant $b=b(\mathcal{C})$ such that

$$
t_{\text {min }}^{\varepsilon} \geq \mu^{-1} \varepsilon^{2}(|\ln \varepsilon|-b)
$$

for all $\varepsilon \in\left(0, \varepsilon_{0}\right)$.

\subsection{Proof of the generation of interface property}

\subsubsection{The bistable ordinary differential equation}

Let us first consider the problem without diffusion, namely

$$
\bar{u}_{t}=\frac{1}{\varepsilon^{2}} f(\bar{u}), \quad \bar{u}(x, 0)=u_{0}(x) .
$$


Its solution can be written in the form

$$
\bar{u}(x, t)=Y\left(\frac{t}{\varepsilon^{2}}, u_{0}(x)\right)
$$

where $Y(\tau, \xi)$ is the solution of the ordinary differential equation

$$
\left\{\begin{array}{l}
Y_{\tau}(\tau, \xi)=f(Y(\tau, \xi)) \quad \text { for } \tau>0 \\
Y(0, \xi)=\xi
\end{array}\right.
$$

Here $\xi$ ranges over the interval $\left(-C_{0}, C_{0}\right)$, where $C_{0}:=\left\|u_{0}\right\|_{L^{\infty}(\Omega)}+1$. We claim that $Y$ has the following properties.

Lemma 7. There exists a positive constant $C$ such that the following holds

(i) If $\xi>0$ then $Y(\tau, \xi)>0$, If $\xi<0$ then $Y(\tau, \xi)<0$;

(ii) $|Y(\tau, \xi)| \leq C_{0}$;

(iii) $0<Y_{\xi}(\tau, \xi) \leq C$;

(iv) $\left|\frac{Y_{\xi \xi}}{Y_{\xi}}(\tau, \xi)\right| \leq C\left(e^{\mu \tau}-1\right)$,

for all $\tau>0$ and all $\xi \in\left(-C_{0}, C_{0}\right)$.

Properties (i) and (ii) are direct consequences of the profile of $f$ - more precisely of the sign conditions $f>0$ in $(-\infty, 0) \cup(a, 1)$ and $f<0$ in $(0, a) \cup(1, \infty)$ - and of the qualitative properties of the solution of the bistable ordinary differential equation (4.5); for proofs of (iii) and (iv) we refer to [2], subsection 3.1 .

\subsubsection{Construction of sub- and super-solutions}

We use the notation $a^{+}=\max (a, 0)$. The sub- and super-solutions are given by

$$
w_{\varepsilon}^{ \pm}(x, t)=\left[Y\left(\frac{t}{\varepsilon^{2}}, u_{0}(x) \pm \varepsilon^{2} C^{\star}\left(e^{\mu t / \varepsilon^{2}}-1\right)\right)\right]^{+} .
$$

Lemma 8. There exist positive constants $\varepsilon_{0}$ and $C^{\star}$ such that, for all $\varepsilon \in\left(0, \varepsilon_{0}\right),\left(w_{\varepsilon}^{-}, w_{\varepsilon}^{+}\right)$is a pair of sub-and super-solutions for Problem $\left(P^{\varepsilon}\right)$, in the domain $\bar{\Omega} \times\left[0, \mu^{-1} \varepsilon^{2}|\ln \varepsilon|\right]$. Moreover, since also $w_{\varepsilon}^{-}(x, 0)=w_{\varepsilon}^{+}(x, 0)=u_{0}(x)$, it follows that

$$
w_{\varepsilon}^{-}(x, t) \leq u^{\varepsilon}(x, t) \leq w_{\varepsilon}^{+}(x, t) \quad \text { for all }(x, t) \in \bar{\Omega} \times\left[0, \mu^{-1} \varepsilon^{2}|\ln \varepsilon|\right] .
$$


Proof. In order to prove that $\left(w_{\varepsilon}^{-}, w_{\varepsilon}^{+}\right)$is a pair of sub- and super-solutions for Problem $\left(P^{\varepsilon}\right)$ for a suitable choice of $\varepsilon_{0}$ and $C^{\star}$, we check that the sufficient conditions in Lemma 3 are satisfied.

As for the sub-solution $w_{\varepsilon}^{-}$, we remak that property (i) in Lemma 7 implies that, for all $t>0$,

$$
\begin{aligned}
& \Omega_{t}^{\text {supp }}\left[w_{\varepsilon}^{-}\right]=\left\{x \in \Omega \mid u_{0}(x)>\varepsilon^{2} C^{\star}\left(e^{\mu t / \varepsilon^{2}}-1\right)\right\} \\
& \Gamma_{t}^{\text {supp }}\left[w_{\varepsilon}^{-}\right]:=\partial \Omega_{t}^{\text {supp }}\left[w_{\varepsilon}^{-}\right] \subset\left\{x \in \Omega \mid u_{0}(x)=\varepsilon^{2} C^{\star}\left(e^{\mu t / \varepsilon^{2}}-1\right)\right\} .
\end{aligned}
$$

Choose $\left(x_{0}, t_{0}\right)$ such that $x_{0} \in \Gamma_{t_{0}}^{\text {supp }}\left[w_{\varepsilon}^{-}\right]$; for $(x, t)$ such that $x \in \Omega_{t}^{\text {supp }}\left[w_{\varepsilon}^{-}\right]$we have

$$
\nabla\left(w_{\varepsilon}^{-}\right)^{m}(x, t)=m Y^{m-1} Y_{\xi}\left(\frac{t}{\varepsilon^{2}}, u_{0}(x)-\varepsilon^{2} C^{\star}\left(e^{\mu t / \varepsilon^{2}}-1\right)\right) \nabla u_{0}(x) .
$$

Since $Y(\tau, 0)=0$ the equality above implies

$$
\lim _{\substack{(x, t) \rightarrow\left(x_{0}, t_{0}\right) \\ x \in \Omega_{t}^{\text {supp }}\left[w_{\varepsilon}^{-}\right]}} \nabla\left(w_{\varepsilon}^{-}\right)^{m}(x, t)=0 .
$$

Therefore conditions (i) and (iii) of Lemma 3 are satisfied by the sub-solution.

As for the super-solution $w_{\varepsilon}^{+}$, we remark that property (i) of Lemma 7 implies that, for all $t>0$

$$
\begin{aligned}
& \Omega_{t}^{\text {supp }}\left[w_{\varepsilon}^{+}\right]=\Omega \\
& \Gamma_{t}^{\text {supp }}\left[w_{\varepsilon}^{+}\right]:=\partial \Omega_{t}^{\text {supp }}\left[w_{\varepsilon}^{+}\right]=\partial \Omega .
\end{aligned}
$$

Hence condition (iii) of Lemma 3 for the super-solution is a direct consequence of the fact that Supp $u_{0} \subset \subset \Omega$, whereas condition (i) is obviously satisfied.

It remains to prove that

$$
\mathcal{L}\left[w_{\varepsilon}^{-}\right]:=\left(w_{\varepsilon}^{-}\right)_{t}-\Delta\left(w_{\varepsilon}^{-}\right)^{m}-\frac{1}{\varepsilon^{2}} f\left(w_{\varepsilon}^{-}\right) \leq 0,
$$

in $\left\{(x, t) \in \bar{\Omega} \times\left[0, \mu^{-1} \varepsilon^{2}|\ln \varepsilon|\right]\right.$ such that $\left.w_{\varepsilon}^{-}(x, t)>0\right\}$ and that $\mathcal{L}\left[w_{\varepsilon}^{+}\right] \geq 0$ in $\{(x, t) \in \bar{\Omega} \times$ $\left.\left[0, \mu^{-1} \varepsilon^{2}|\ln \varepsilon|\right]\right\}$.

In view of the ordinary differential equation (4.5), straightforward computations yield

$$
\begin{aligned}
\mathcal{L}\left[w_{\varepsilon}^{-}\right]=-Y_{\xi}\left[C^{\star} \mu e^{\mu t / \varepsilon^{2}}+m(m-1) Y^{m-2} Y_{\xi}\left|\nabla u_{0}\right|^{2}\right. & \\
& \left.+m Y^{m-1} \frac{Y_{\xi \xi}}{Y_{\xi}}\left|\nabla u_{0}\right|^{2}+m Y^{m-1} \Delta u_{0}\right],
\end{aligned}
$$

in $\Omega_{t}^{\text {supp }}\left[w_{\varepsilon}^{-}\right]$, where the function $Y$ and its derivatives are taken at the point $(\tau, \xi)=\left(t / \varepsilon^{2}, u_{0}(x)-\right.$ $\left.\varepsilon^{2} C^{\star}\left(e^{\mu t / \varepsilon^{2}}-1\right)\right)$. Moreover since the term $m(m-1) Y^{m-2} Y_{\xi}^{2}\left|\nabla u_{0}\right|^{2}$ is nonnegative, it follows that

$$
\mathcal{L}\left[w_{\varepsilon}^{-}\right] \leq-Y_{\xi}\left[C^{\star} \mu e^{\mu t / \varepsilon^{2}}+m Y^{m-1} \frac{Y_{\xi \xi}}{Y_{\xi}}\left|\nabla u_{0}\right|^{2}+m Y^{m-1} \Delta u_{0}\right] .
$$


We note that, in the range $0 \leq t \leq \mu^{-1} \varepsilon^{2}|\ln \varepsilon|$, we have, for $\varepsilon_{0}$ sufficiently small,

$$
\xi=u_{0}(x)-\varepsilon^{2} C^{\star}\left(e^{\mu t / \varepsilon^{2}}-1\right) \in\left(-C_{0}, C_{0}\right) .
$$

We deduce from the properties (ii)-(iv) stated in Lemma 7 that there exist positive constants $C_{1}$ and $C_{2}$ - only depending on $m, C_{0}, C,\left\|\nabla u_{0}\right\|_{L^{\infty}(\Omega)}$ and $\left\|\Delta u_{0}\right\|_{L^{\infty}(\Omega)}$ - such that

$$
\mathcal{L}\left[w_{\varepsilon}^{-}\right] \leq-Y_{\xi}\left[\left(C^{\star} \mu-C_{1}\right) e^{\mu t / \varepsilon^{2}}-C_{2}\right],
$$

which implies that $\mathcal{L}\left[w_{\varepsilon}^{-}\right] \leq 0$ if $C^{\star}$ is chosen large enough.

As for the super-solution we obtain

$$
\begin{aligned}
\mathcal{L}\left[w_{\varepsilon}^{+}\right]=Y_{\xi}\left[C^{\star} \mu e^{\mu t / \varepsilon^{2}}-m(m-1) Y^{m-2} Y_{\xi}\left|\nabla u_{0}\right|^{2}\right. & \\
& \left.-m Y^{m-1} \frac{Y_{\xi \xi}}{Y_{\xi}}\left|\nabla u_{0}\right|^{2}-m Y^{m-1} \Delta u_{0}\right],
\end{aligned}
$$

and the assumption that $m \geq 2$ gives an upper bound for $\left|Y^{m-2}\right|$. Following the same argument as above one can prove that $\mathcal{L}\left[w_{\varepsilon}^{+}\right] \geq 0$ for $C^{\star}$ sufficiently large. This completes the proof of Lemma 8.

\subsubsection{Proof of Theorem 4}

In order to prove Theorem 4 we first present basic estimates of the function $Y$ after a time of order $\tau \sim|\ln \varepsilon|$.

Lemma 9. Let $\gamma \in(0, \min (a, 1-a))$ be arbitrary. There exist positive constants $\varepsilon_{0}$ and $C_{Y}$ such that, for all $\varepsilon \in\left(0, \varepsilon_{0}\right)$,

(i) for all $\xi \in\left(-C_{0}, C_{0}\right)$,

$$
-\gamma \leq Y\left(\mu^{-1}|\ln \varepsilon|, \xi\right) \leq 1+\gamma
$$

(ii) for all $\xi \in\left(-C_{0}, C_{0}\right)$ such that $|\xi-a| \geq C_{Y} \varepsilon$, we have that

$$
\begin{aligned}
& \text { if } \xi \geq a+C_{Y} \varepsilon \text { then } Y\left(\mu^{-1}|\ln \varepsilon|, \xi\right) \geq 1-\gamma \\
& \text { if } \xi \leq a-C_{Y} \varepsilon \text { then } Y\left(\mu^{-1}|\ln \varepsilon|, \xi\right) \leq \gamma .
\end{aligned}
$$

These estimates illustrate the stability of the equilibria 0 and 1 for the bistable ordinary differential equation (4.5). For more details we refer the reader to the proof of Lemma 3.9 in [2].

We are now ready to prove Theorem 4. By setting $t=\mu^{-1} \varepsilon^{2}|\ln \varepsilon|$ in (4.7), we obtain

$$
\begin{aligned}
{\left[Y \left(\mu^{-1}|\ln \varepsilon|, u_{0}(x)\right.\right.} & \left.\left.-\left(C^{\star} \varepsilon-C^{\star} \varepsilon^{2}\right)\right)\right]^{+} \\
& \leq u^{\varepsilon}\left(x, \mu^{-1} \varepsilon^{2}|\ln \varepsilon|\right) \leq\left[Y\left(\mu^{-1}|\ln \varepsilon|, u_{0}(x)+C^{\star} \varepsilon-C^{\star} \varepsilon^{2}\right)\right]^{+} .
\end{aligned}
$$


Since, for $\varepsilon_{0}$ small enough, $u_{0}(x)+\left(C^{\star} \varepsilon-C^{\star} \varepsilon^{2}\right) \in\left(0, C_{0}\right)$, the assertion $(i)$ of Theorem 4 is a direct consequence of (4.10) and (4.13).

Next we prove (4.2). We choose $M_{0}$ large enough so that $M_{0} \varepsilon-C^{\star} \varepsilon+C^{\star} \varepsilon^{2} \geq C_{Y} \varepsilon$. Then, for all $x \in \Omega$ such that $u_{0}(x) \geq a+M_{0} \varepsilon$, we have that $u_{0}(x)-\left(C^{\star} \varepsilon-C^{\star} \varepsilon^{2}\right) \geq a+C_{Y} \varepsilon$, which we combine with (4.13) and (4.11) to deduce that

$$
u^{\varepsilon}\left(x, \mu^{-1} \varepsilon^{2}|\ln \varepsilon|\right) \geq 1-\gamma .
$$

The inequality (4.3) can be shown in a similar way. This completes the proof of Theorem 4.

Remark 10. Theorem 4 remains true if we perturb the reaction function $f(u)$ by order $\varepsilon$, setting for instance $\tilde{f}(u)=f(u)-\varepsilon g(x, t, u)$. To deal with this more general case, we proceed as follows. We first consider a slightly perturbed reaction function, namely $f_{\delta}(u)=f(u)+\delta$ which, for $\delta$ small enough, is still of bistable type. Define a $(\delta)$ as its unstable zero and $\mu(\delta):=f^{\prime}(a(\delta))$ as the slope of $f_{\delta}$ in this point. We then define $Y(\tau, \xi ; \delta)$ as the solution of the initial value problem

$$
\left\{\begin{array}{l}
Y_{\tau}(\tau, \xi ; \delta)=f_{\delta}(Y(\tau, \xi ; \delta)) \quad \text { for } \tau>0 \\
Y(0, \xi ; \delta)=\xi .
\end{array}\right.
$$

Finally we construct a pair of sub-and super-solutions in the form

$$
w_{\varepsilon}^{ \pm}(x, t)=\left[Y\left(\frac{t}{\varepsilon^{2}}, u_{0}(x) \pm \varepsilon^{2} r\left( \pm \varepsilon \mathcal{G}, \frac{t}{\varepsilon^{2}}\right) ; \pm \varepsilon \mathcal{G}\right)\right]^{+}
$$

where $r(\delta, \tau):=C^{\star}\left(e^{\mu(\delta) \tau}-1\right)$ and $\mathcal{G}:=\|g\|_{L^{\infty}\left(0, C_{0}\right)}$. For more details and proofs we refer to [2], more precisely to Section 4 which deals with a generation of interface property for an equation with linear diffusion and an unbalanced reaction term $f(u)$.

\subsection{Proof of the optimality of the generation time}

We show below that the generation time $t^{\varepsilon}:=\mu^{-1} \varepsilon^{2}|\ln \varepsilon|$ is optimal. In other words, the interface is not fully developed before $t$ is close to $t^{\varepsilon}$. We will need the following lemma about the solution $Y(\tau, \xi)$ of the corresponding bistable ordinary differential equation.

Lemma 11. Let $\eta \in(0, \min (a, 1-a))$ be arbitrary. Then there exist positive constants $C_{1}=C_{1}(\eta)$ and $C_{2}=C_{2}(\eta)$ such that, if $\xi \in(a, 1-\eta)$ then, for every $\tau>0$ such that $Y(\tau, \xi)$ remains in the interval $(a, 1-\eta)$, we have

$$
C_{1} e^{\mu \tau}(\xi-a) \leq Y(\tau, \xi)-a \leq C_{2} e^{\mu \tau}(\xi-a) .
$$

We refer to [2] Corollary 3.5 for the proof of Lemma 11.

Proof of Proposition 6. For each $b>0$, we set

$$
t^{\varepsilon}(b):=\mu^{-1} \varepsilon^{2}(|\ln \varepsilon|-b),
$$


and evaluate $u^{\varepsilon}\left(x, t^{\varepsilon}(b)\right)$ at a point $x \in \Omega_{0}^{(1)}$ such that $\operatorname{dist}\left(x, \Gamma_{0}\right)=\mathcal{C} \varepsilon$. Define $M:=\left\|u_{0}\right\|_{C^{2}(\bar{\Omega})}$. Since $u_{0}=a$ on $\Gamma_{0}$, we have that

$$
u_{0}(x) \leq a+M \mathcal{C} \varepsilon,
$$

which implies together with Lemma 11 that

$$
\begin{aligned}
w_{\varepsilon}^{+}\left(x, t^{\varepsilon}(b)\right) & =Y\left(\mu^{-1}(|\ln \varepsilon|-b), u_{0}(x)+\varepsilon C^{\star} e^{-b}-\varepsilon^{2} C^{\star}\right) \\
& \leq a+C_{2} e^{|\ln \varepsilon|-b}\left(u_{0}(x)+\varepsilon C^{\star} e^{-b}-\varepsilon^{2} C^{\star}-a\right) \\
& \leq a+C_{2} \varepsilon^{-1} e^{-b}\left(M \mathcal{C} \varepsilon+\varepsilon C^{\star} e^{-b}\right) \\
& =a+C_{2} e^{-b}\left(M \mathcal{C}+C^{\star} e^{-b}\right) .
\end{aligned}
$$

Now choose $b$ large enough so that

$$
a+C_{2} e^{-b}\left(M \mathcal{C}+C^{\star} e^{-b}\right)<1-\eta
$$

the inequalities (4.17) and (4.7) then yield

$$
u^{\varepsilon}\left(x, t^{\varepsilon}(b)\right) \leq w_{\varepsilon}^{+}\left(x, t^{\varepsilon}(b)\right)<1-\eta .
$$

Therefore (4.4) does not hold at $t=t^{\varepsilon}(b)$, and hence $t^{\varepsilon}(b)<t_{m i n}^{\varepsilon}$. This completes the proof of Proposition 6.

\section{References}

[1] M. Alfaro. The singular limit of a chemotaxis-growth system with general initial data. Adv. Differential Equations, 11 (2006), no. 11, 1227-1260.

[2] M. Alfaro, D. Hilhorst, H. Matano. The singular limit of the Allen-Cahn equation and the FitzHugh-Nagumo system. J. Differential Equations, 245 (2008), 505-565.

[3] D. Aronson, M. G. Crandall, L. A. Peletier. Stabilization of solutions of a degenerate nonlinear diffusion problem. Nonlinear Anal., 6 (1982), 1001-1022.

[4] E. A. Carl. Population control in arctic ground squirrels. Ecology, 52 (1971), 395-413.

[5] X. Chen. Generation and propagation of interfaces for reaction-diffusion equations. J. Differential Equations, 96 (1992), 116-141.

[6] X. Chen. Generation and propagation of interfaces for reaction-diffusion systems. Trans. Amer. Math. Soc., 334 (1992), 877-913.

[7] E. DiBenedetto. Continuity of weak solutions to a general porous medium equation. Indiana University Mathematics J., 32 (1983), 83-118. 
[8] E. Feireisl. Front propagation for degenerate parabolic equations. Nonlinear Anal., 35 (1999), 735-746.

[9] W. S. C. Gurney, R. M. Nisbet. The regulation of inhomogeneous populations. J. Theoret. Biol., 52 (1975), 441-457.

[10] M. E. Gurtin, R. C. MacCamy. On the diffusion of biological populations. Math. Biosci., 33 (1979), 35-49.

[11] D. Hilhorst, R. Kersner, E. Logak, M. Mimura. Interface dynamics of the Fisher equation with degenerate diffusion. J. Differential Equations, 244 (2008), 2872-2889.

[12] J. L. Vásquez. The porous medium equation. Mathematical theory. Oxford University Press, Oxford, 2007. 